\title{
CoDesign with people living with cognitive and sensory impairments
}

\section{Karin Slegers, Pieter Duysburgh \& Niels Hendriks}

To cite this article: Karin Slegers, Pieter Duysburgh \& Niels Hendriks (2015) CoDesign with people living with cognitive and sensory impairments, CoDesign, 11:1, 1-3, DOI: 10.1080/15710882.2015.1020102

To link to this article: http://dx.doi.org/10.1080/15710882.2015.1020102

\section{曲 Published online: 24 Mar 2015.}

Submit your article to this journal

Џ Article views: 189

Q View related articles $₫$

View Crossmark data \ 


\section{EDITORIAL}

\section{CoDesign with people living with cognitive and sensory impairments}

This special issue aims to bring together current research on codesign with people living with cognitive or sensory impairments. The idea for this special issue grew from the challenges the guest editors encountered in their own codesign projects involving such end-users. These challenges are at least partly related to the fact that people living with cognitive or sensory impairments may make sense of the world, and may share their experiences in a radically different way than other end-users and codesign researchers and designers themselves. As a result, researchers, designers and codesign participants living with impairments may find it difficult to understand each other, and to communicate. In addition, participants' impairments may pose challenges on a methodological level. For instance, many cognitive and sensory impairments are related to thought processes and communication skills, including memory, sequencing actions, understanding abstractions and interpreting social cues. These are precisely the processes and skills that many participatory and codesign techniques draw upon. Therefore, such techniques might not be usable at all, or may need to be adjusted for working with people with impairments.

In our view, an important prerequisite for codesign is to create a 'space' for common understanding and creation. This can be understood as a 'hybrid space' where a process of mutual learning and shaping can occur. The concept of such a hybrid space, as defined by Muller (2003), serves as an in-between region for researchers and designers on the one hand and end-users on the other hand. It is an overlap between two different worlds and is not owned by the researcher, the designer or by the end-user. As such, this hybrid space is open to differences and thus may serve as a place for poly-vocal discussion and co-creation: a bridge between the world of (technology) developers, designers and researchers on the one hand, and the world of end-users on the other. When working with participants living with a cognitive or sensory impairment, the task of creating a hybrid space may require more thought and preparation than for other codesign projects. Therefore, in the call for papers for this special issue, we asked the design research community to report on the methods, techniques and tools they use in codesign in order to create hybrid spaces for designing with persons with cognitive or sensory impairments. More specifically, we asked for papers dealing with methodological issues, such as the customisation of existing codesign techniques to make them suitable for use with participants living with impairments.

When adjusting codesign techniques for users living with cognitive or sensory impairments, a critical issue seems to be that different adjustments have to be devised for specific user groups. Unfortunately, the reasoning that informed the adjustments that have been reported until now has not always been clearly described. As a result, it is difficult for researchers and designers to generalise the lessons learned from previous adjustments to future research. This special issue examines whether it is possible to extract general guidelines for codesigning with end-users living with cognitive or sensory impairments. Do researchers and designers who do codesign with people living with cognitive or sensory impairments need a different set of guidelines than designers who work with other types of users? 
The authors of the five papers in this special issue all have examined this question through their explorative and experimental methodological approaches. They have attempted to go beyond using proxies as informants and looked for ways to have persons living with an impairment directly participate in the design process themselves. Despite the theoretical underpinnings of their research, finding suitable approaches was challenging for all of the authors.

In the first paper, Brereton et al. argue that involving proxies (professional carers, family) alone is insufficient in design research: despite their high involvement, their knowledge of the user living with a cognitive or sensory impairment is limited and often biased. Also, much of the use of a future product is unanticipated: end-users always have their own way of making use of a product. In two case studies, the authors clearly illustrate this process of 'design after design' or 'design in use' in the context of working with persons living with impairments. By working with simple, concrete and functional prototypes, the exploration of such unanticipated use was facilitated, thus informing designers on further design choices.

Wilson et al., in the second paper, report on their codesign activities with people with aphasia. When developing projects on gesture therapy and a virtual world for practicing speech, they made use of a myriad of methods to inform the design when exploring the issue of tangibility in fostering hybridity. The authors make clear that their choice of methods was more influenced by serendipity than by strategic planning. While the impact of the tools they used cannot be quantified, their experiences with tangible design language are instructive and often counterintuitive. For instance, they advocate the use of highfidelity prototypes (as opposed to the low-fidelity prototypes that are often preferred in literature on doing codesign) since there are less abstract and result in more useful feedback from users with aphasia.

Metatla et al. also explored the issue of tangibility in their research with users living with visual impairments. More particularly, they examine cross-modal interaction by making use of audio-haptic objects to understand how visual representations may be replaced by making use of sound or tangible elements. Remarkably, constructing the audio-haptic mock-ups in the initial, explorative phase of the research seemed to hinder rather than facilitate communication between end-users and researchers and designers. This resulted in a negative effect from visual mock-ups, which are commonly used to encourage interaction between both parties. However, in the prototyping phase, the high malleability of the prototypes did allow for easy production of alternative representations of a given functionality, which then resulted in a successful involvement of the participants living with visual impairments.

Gaudion et al. advocate a bottom-up, phenomenological approach, in which adults living with autism spectrum disorder describe their experiences and their environments to inform design to create spaces, objects and activities that are more meaningful to them. The authors make use of abstract props, sensory preference cards, objects of everyday use, and other approaches to configure design activities that are not driven by preselected methods with specific aims or goals. Their studies entirely evolved through the developing understanding of the researcher. They conclude that rather than developing autismfriendly design methods, engagement should concentrate on how information is derived, interpreted and disseminated.

Finally, Hendriks et al. further explore the search for a specific approach for doing codesign with people living with cognitive or sensory impairments. They ask whether it is really feasible to work towards a specific approach for such end-user groups or whether each codesign activity is, in essence, so unique that general guidelines are fruitless. Based 
on a series of academic workshops on the topic and a literature review, the authors have come to favour an approach of sharing method stories over the search for general guidelines for doing codesign with people living with cognitive or sensory impairments. Their experience leads them to argue that providing insights into the reasoning behind methodological decisions and reporting on successes and failures is more valuable for others than attempting to provide general guidelines.

This special issue highlights the different approaches that researchers and designers have put forward in working with persons living with cognitive or sensory impairments. With this overview of approaches, we aim to contribute to the ongoing discussion about how to involve persons living with cognitive or sensory impairments in the design process.

In conclusion, we express our gratitude to all authors and reviewers who were involved in the selection process of the papers in this issue. We also thank the Editor-in-Chief, Prof. Janet McDonnell, for her co-operation and support throughout the creation of this special issue.

\section{References}

Muller, Michael, J. 2003. "Participatory Design: The Third Space in HCI." In The HumanComputer Interaction Handbook, edited by Michael J. Muller, Julie A. Jacko, and Andrew Sears, 1051-1068. Hillsdale, NJ: L. Erlbaum Associates. http://dl.acm.org/citation.cfm?id=772072. 772138

Karin Slegers

CUO | Social Spaces, KU Leuven - iMinds, Leuven, Belgium

Pieter Duysburgh

SMIT, VUB - iMinds, Brussels, Belgium

Niels Hendriks Social Spaces | CUO, LUCA School of Arts - KU Leuven, Genk, Belgium 